\section{Entschließung der Konferenz der Datenschutzbeauftragten des Bundes und der Länder vom 05. September 2013}

Keine umfassende und anlasslose Überwachung durch Nachrichtendienste! Zeit für Konsequenzen

Die Konferenz der Datenschutzbeauftragten des Bundes und der Länder stellt fest, dass noch immer nicht alles getan wurde, um das Ausmaß der nachrichtendienstlichen Ermittlungen mithilfe von Programmen wie PRISM, TEMPORA und XKEYSCORE für die Bundesrepublik Deutschland aufzuklären.

Schon die bisherigen Erkenntnisse lassen den Schluss zu, dass die Aktivitäten u.a. des US-amerikanischen und des britischen Geheimdienstes auf eine globale und tendenziell unbegrenzte Überwachung der Internetkommunikation hinauslaufen, zumal große Internet- und Telekommunikationsunternehmen in die Geheimdienstaktionen eingebunden sind.

Da zahlreiche Anbieter von Kommunikationsdienstleistungen, deren Server in den USA stehen, personenbezogene Daten der Menschen in der Bundesrepublik Deutschland verarbeiten, betreffen die Berichte, dass US-amerikanische Geheimdienste auf dem Territorium der USA personenbezogene Daten umfassend und anlasslos überwachen, auch ihre Daten. Unklar ist daneben noch immer, ob bundesdeutsche Stellen anderen Staaten rechtswidrig personenbezogene Daten für deren Zwecke zur Verfügung gestellt und ob bundesdeutsche Stellen rechtswidrig erlangte Daten für eigene Zwecke genutzt haben.

Die staatliche Pflicht zum Schutz der Grundrechte erfordert es, sich nicht mit der gegenwärtigen Situation abzufinden. Die Regierungen und Parlamente des Bundes und der Länder sind dazu aufgerufen, das ihnen im Rahmen ihrer Zuständigkeiten Mögliche zu tun, um die Einhaltung des deutschen und des europäischen Rechts zu gewährleisten. Das Bundesverfassungsgericht hat festgestellt, dass es „zur verfassungsrechtlichen Identität der Bundesrepublik Deutschland gehört, für deren Wahrung sich die Bundesrepublik in europäischen und internationalen Zusammenhängen einsetzen muss", "dass die Freiheitswahrnehmung der Bürger nicht total erfasst und registriert werden darf". Es müssen daher alle Maßnahmen getroffen werden, die den Schutz der informationellen Selbstbestimmung der in Deutschland lebenden Menschen und ihr Grundrecht auf Vertraulichkeit und Integrität informationstechnischer Systeme für die Zukunft sicherstellen.

Für die Wahrung der Grundrechte der Menschen in der Bundesrepublik Deutschland kommt es nun darauf an, die notwendigen Konsequenzen zu ziehen.

Die Konferenz der Datenschutzbeauftragten des Bundes und der Länder fordert deshalb:

- Nationales, europäisches und internationales Recht so weiterzuentwickeln und umzusetzen, dass es einen umfassenden Schutz der Privatsphäre, der informationellen Selbstbestimmung, des Fernmeldegeheimnisses und des Grundrechts auf Vertraulichkeit und Integrität informationstechnischer Systeme garantiert.
- Sofern verfassungswidrige nachrichtendienstliche Kooperationen erfolgen, müssen diese abgestellt und unterbunden werden.

- Die Kontrolle der Nachrichtendienste muss durch eine Erweiterung der Befugnisse sowie eine gesetzlich festgelegte verbesserte Ausstattung der parlamentarischen Kontrollgremien intensiviert werden. Bestehende Kontrollücken müssen unverzüglich geschlossen werden. In diesem Zusammenhang ist zu prüfen, ob die Datenschutzbeauftragten verstärkt in die Kontrolle der Nachrichtendienste eingebunden werden können.

- Es sind Initiativen zu ergreifen, die die informationelle Selbstbestimmung und das Grundrecht auf Vertraulichkeit und Integrität informationstechnischer Systeme sicherstellen. Dazu gehört, - zu prüfen, ob das Routing von Telekommunikationsverbindungen in Zukunft möglichst nur über Netze innerhalb der EU erfolgen kann.

- sichere und anonyme Nutzungsmöglichkeiten von Telekommunikationsangeboten aller Art auszubauen und zu fördern. Dabei ist sicherzustellen, dass den Betroffenen keine Nachteile entstehen, wenn sie die ihnen zustehenden Rechte der Verschlüsselung und Nutzung von Anonymisierungsdiensten ausüben.

- die Voraussetzungen für eine objektive Prüfung von Hard-und Software durch unabhängige Zertifizierungsstellen zu schaffen.

- Völkerrechtliche Abkommen wie das Datenschutz-Rahmenabkommen und das Freihandelsabkommen zwischen der EU und den USA dürfen nur abgeschlossen werden, wenn die europäischen Datenschutzgrundrechte ausreichend geschützt werden. Das bedeutet auch, dass jeder Mensch das Recht hat, bei vermutetem Datenmissbrauch den Rechtsweg zu beschreiten. Das Fluggastdatenabkommen und das Überwachungsprogramm des Zahlungsverkehres müssen auf den Prüfstand gestellt werden.

- Auch innerhalb der Europäischen Union ist sicherzustellen, dass die nachrichtendienstliche Überwachung durch einzelne Mitgliedstaaten nur unter Beachtung grundrechtlicher Mindeststandards erfolgt, die dem Schutzniveau des Art. 8 der Charta der Grundrechte der Europäischen Union entsprechen.

Die Konferenz der Datenschutzbeauftragten des Bundes und der Länder fordert alle Verantwortlichen auf, die umfassende Aufklärung mit Nachdruck voranzutreiben und die notwendigen Konsequenzen zügig zu treffen. Es geht um nichts weniger als das Grundvertrauen der Bürgerinnen und Bürger in den Rechtsstaat.

\section{Gl zur Spähaffaire: Informatiker klären auf}

Die Ausspähung durch Nachrichtendienste beherrscht die Medien; Fach- und Sachaufklärung kommen aus Sicht vieler Informatikerinnen und Informatiker dabei zu kurz. Deshalb hat ein Arbeitskreis der Gesellschaft für Informatik e.V. (GI) unter Leitung von GI-Vizepräsidentin Simone Rehm eine Liste von knapp 40 Fragen und Ant- 